Pacific Journal of Mathematics

SPANNING SURFACES FOR PROJECTIVE PLANES IN FOUR 


\title{
SPANNING SURFACES FOR PROJECTIVE PLANES IN FOUR SPACE
}

\author{
T. M. PrICE
}

The purpose of this paper is to investigate two questions about the complements of projective planes in $S^{4}$. We work in the piecewise linear (PL) category (see Hudson [7] for basic definitions). All embeddings are assumed to be PL locally flat. (Equivalently PL locally unknotted, see Hudson [7] page 138.) We note though, that P.L locally flat codimension two embeddings are smoothable, and vice versa (see the paragraph preceding Lemma 1) so that we could work with smooth embeddings instead. The first problem we consider is the bordism problem. Of course, we are immediately faced with the well-known fact that the projective plane does not bound any 3-manifold. Progress is further hampered by Whitney's result, [14], that a smooth projective plane in $E^{4}$ does not support a normal vector field. It follows that a PL locally flat projective plane in $S^{4}$ cannot lie on the boundary of a 3 -manifold in $S^{4}$ nor can it lie in the interior of a 3-manifold in $S^{4}$. The solution to this dilemma lies in the concept of a 3-manifold with singular points. We show, Theorem 2, that every PL locally flat projective plane in $S^{4}$ bounds a 3-manifold with singular points in $S^{4}$. We also show, Theorem 1 , that a PL locally flat projective plane in $S^{4}$ is unknotted iff it bounds a particular 3-manifold with singular points (namely, the cone over a Moebius band). The second problem we investigate here is a mapping problem; namely, does the complement of a knotted projective plane map onto the complement of the unknotted projective plane. In Theorem 3 we give a necessary and sufficient condition for this to occur. While the condition in Theorem 3 is necessary and sufficient, we feel there are better results possible and we discuss the shortcomings of Theorem 3 and conjecture a better result.

The first theorem characterizes unknotted projective planes in a fashion analogous to the result that a PL locally flat 2 -sphere in $S^{4}$ is unknotted iff it bounds a PL 3-cell. First we remark that the standard or cannonical projective plane in $S^{4}$, denoted $p^{2}$, is the one sketched in Fig. 1 and described just before Lemma 1. (See Price and Roseman [10] for a more complete discussion of this choice of cannonical embedding.) Analogous to the case for spheres, we define a PL locally flat projective plane $P$, in $S^{4}$ to be unknotted iff there exists a PL homeomorphism $h: S^{4} \rightarrow S^{4}$ with $h(P)=P^{2}$. To describe the analogue of the 3-cell we need the concept of a cone. Briefly, 
if $X$ is a subset of $S^{3} \supseteqq S^{4}$ and $v$ is a point in $S^{4}-S^{3}$, then the cone over $X$ from $v$, denoted $v * X$, is the union of all "straight line segments" starting at $v$ and ending in $X$ (see page 6 of Hudson [7] for a definition of "join", which is a generalization of "cone"). In particular, if $M^{2}$ is a Moebius band in $S^{3}$ and if $v \in S^{4}-S^{3}$, then we are interested in $v * M^{2}$. In this case $M^{2} \cup v *\left(\partial M^{2}\right) \subseteq v * M^{2}$ is homeomorphic to $p^{2}$ and is referred to as the boundary of $v * M^{2}$, denoted $\partial\left(v * M^{2}\right)$. We say that a projective plane $P$ in $S^{4}$ bounds a polyhedron, $R$, with PL homeomorphic to the cone over a Moebius band iff there exists a PL embidding $h: v * M^{2} \rightarrow S^{4}$ such that $P=h\left(\partial\left(v * M^{2}\right)\right)$.

THeORem 1. Let $P$ be a $P L$ locally flat projective plane in $S^{4}$. Then $P$ is unknotted iff $P$ bounds a polyhedron, $R$, with $R P L$ homeomorphic to the cone over a Moebius band.

Proof. It is easy to see from Fig. 1 (by isotoping $M^{2}$ vertically until it lies in $S^{3}=\partial B^{4}$ ) that the cannonical embedding, $p^{2}$, bounds a polyhedron homeomorphic to the cone over a Moebius band. Hence any unknotted projective plane in $S^{4}$ bounds such a polyhedron.

Conversely, suppose $P$ bounds such a polyhedron, $R$. Let $h: v * M^{2} \rightarrow R$ be a PL homeomorphism. Since $v * M^{2}$ collapses to $v$, it is easy to show (e.g., using Lemma 1.22 page 33 and the ideas of $\S 3$ Chapter VI of Hudson, [7]) that there is a PL homeomorphism $H: S^{4} \rightarrow S^{4}$ with $H \circ h: v * M^{2} \rightarrow S^{4}$ being a cone embedding (that is $\left.H \circ h\left(v * M^{2}\right)=(H \circ h(v)) *\left(H \circ h\left(M^{2}\right)\right)\right)$. To simplify notation, we assume that $P=\partial\left(v * M^{2}\right)$ with $v * M^{2}$ embedded in $S^{4}$ as a cone. Let $N$ be a regular neighborbood of $v * M^{2} \bmod M^{2}$ in $S^{4}$ (see Cohen [1]). Then $N$ is a 4-cell and $(\partial N) \cap\left(v * M^{2}\right)=(\partial N) \cap P=M^{2}$ is a Moebius band. Let $J$ be the center line of $M^{2}$. We wish to show that $J$ is unknotted in $\partial N$. Since $P$ is PL locally flat in $S^{4}$ it follows that $v * \partial M^{2}$ (which is a subcone of $v * M^{2} \subseteq N$ ) must be unknotted in $N$ and hence $\partial M^{2}$ must be unknotted in $\partial N$. But $\partial M^{2}$ is a $(2, q)$ cable about $J$ (that is $\partial M^{2}$ lies on the boundary of a solid torus $T$ whose centerline is $J$ and $\partial M^{2}$ goes around $T 2$ times longitudinally and $q$ times meridionally). Since $\partial M$ is unknotted it follows from Schubert Satz 1 page 247 [11] that $0=$ genus $\partial M^{2} \geqq 2$ genus $J$ where the genus of a simple closed curve, $K$, is the smallest integer $n$ for which $K$ bounds on orientable surface with exactly $n$-handles. Hence $J$ bounds a disk and must be unknotted also. Furthermore, since $J$ is unknotted in $\partial N$ we have that $\partial M^{2}$ is a $(2, q)$-torus knot and hence its Alexander polynomial is $\left(t^{2} q-1\right)(t-1) /\left(t^{2}-1\right)(t q-1)$, see Crowell and Fox [2] pages 92, 132. On the other hand $\partial M^{2}$ bounds a locally flat disk in $N$, namely $v * \partial M^{2}$ so it is a slice knot and must have an Alexander polynomial of the form $p(t) p(1 / t)$ for some 
polynomial with integer coefficients, see Fox and Milnor [3] or Terasaka [12]. It follows easily that $q= \pm 1$. Since $\partial M^{2}$ is a $(2, \pm 1)$-torus knot it is now straightforward to construct a PL homeomorphism taking $M^{2}$ onto a standard Moebius band in $\partial N$ and then to show that $P$ is unknotted.

Theorem 1 suggests the following class of polyhedra to be used in bordism problems involving projective planes. A 3-manifold with singular points is a compact polyhedron $X$ in which there exists a finite number of points $x_{1}, x_{2}, \cdots, x_{n}$ with $X-\left\{x_{1}, x_{2}, \cdots, x_{n}\right\}$ being a 3-manifold-with-boundary and each $x_{i}(i=1,2, \cdots, n)$ having a polyhedral neighborhood $N_{i}$, in $X$, satisfying:

(a) $N_{i} \cap$ closure $\left(X-N_{i}\right)=\Sigma_{i}$ is connected 2-manifold with nonempty, connected boundary, and

(b) $N_{i} \approx x_{i} * \Sigma_{i}$.

The $x_{i}$ are called singular points. Furthermore, if $X$ is a 3-manifold with singular points, then we define the interior of $X$ by int $X=$ $\left\{x \in X \mid x\right.$ has a neighborhood, in $X$, homeomorphic to $\left.E^{3}\right\}$ and we define the boundary of $X$ by $\partial X=X-\operatorname{int} X$. We note that $X=$ closure (int $X$ ), that int $X$ is a 3 -manifold, with no boundary and that $\partial X$ is a compact 2-manifold with no boundary. Clearly $v * M^{2}$ is a 3-manifold with singular points and $\partial\left(v * M^{2}\right)=M^{2} \cup v *\left(\partial M^{2}\right)$. Any 3-manifold with singular points can be constructed by starting with an appropriate compact 3 -manifold-with-boundary, $M$, choosing pairwise disjoint connected surfaces $\Sigma_{i}(i=1,2, \cdots, n)$ on $\partial M$ (with $\partial \Sigma_{i}$ being a simple closed curve) and forming $M \bigcup_{\Sigma_{1}} \Sigma_{1} * v_{1} \bigcup_{\Sigma_{2}} \Sigma_{2} *$ $v_{2} \cup \cdots \mathrm{U}_{\Sigma_{n}} \Sigma_{n} * v_{n}$.

In order to simplify the statements and proofs of the next two results we introduce the following notation. We consider $S^{4}$ as being the union of two 4-cells, $B^{4}$ and $B_{0}^{4}$, sharing a common boundary. We consider the standard projective plane, $P^{2}$, in $S^{4}$ as the union of a Moebius band, $M^{2}$, embedded in $B^{4}$ (with $M^{2}$ isotopic, keeping $\partial M^{2}$ fixed, to an unknotted, untwisted Moebius band in $\partial B^{4}$ ) and a disk, $D^{2}$ (namely, the cone over $\partial M^{2}$ ), embedded in $B_{0}^{4}$ (see Fig. 1). For a more complete description of this embedding and a discussion of how this embedding is related to other natural candidates for a standard embedding, see Price and Roseman [10]. The normal disk bundle of $P^{2}$ in $S^{4}$, denoted $\nu\left(P^{2}\right)$, is the union of the normal disk bundle of $M^{2}$ in $B^{4}$, denoted $\nu\left(M^{2}\right)$, and the normal disk bundle of $D^{2}$ in $B_{i}^{4}$, denoted $\nu\left(D^{2}\right)$. We identify $\nu\left(M^{2}\right)$ with the mapping cylinder of the 2 to 1 convering map of the solid torus, $T^{3}$, onto the solid Klein bottle, $K^{3}$ (see Fig. 1). We let $a$ denote the boundary of one of the meridional disks of $K^{3}$ and we call any curve isotopic to $a$, in $\partial \nu\left(P^{2}\right)$, a meridian of $\partial \nu\left(P^{2}\right)$. We let $b$ denote a simple closed 
curve on $K^{2}=\partial K^{3}$ that intersects some meridional disk exactly once (transversally) and that bounds a disk in $B^{4}-$ int $\nu\left(M^{2}\right)$ (see Fig. 1). We call $b$ the standard longitude on $\partial \nu\left(P^{2}\right)$.

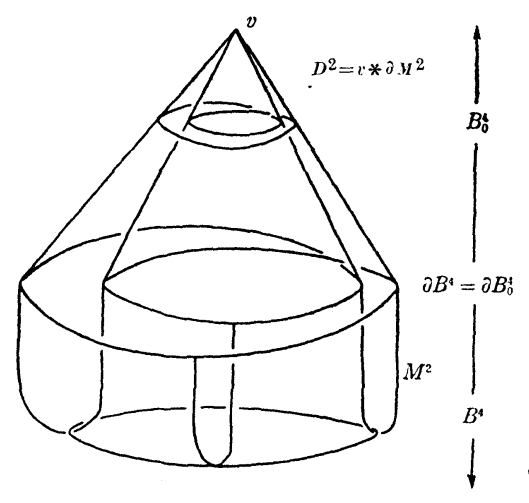

$P^{2}=$ standard projective plane

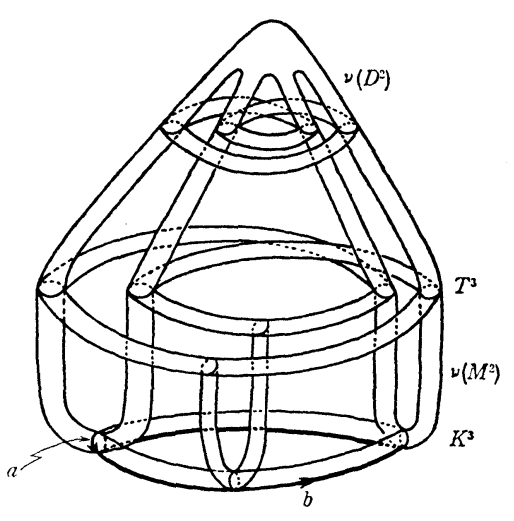

$\nu\left(P^{2}\right)=$ normal disk bundle

Figure 1

Let $P$ be an arbitrary PL locally flat embedding of the projective plane in $S^{4}$. Using results of Wall [13] and Hirsch [5] we can ambient isotope $P$ to a smooth embedding taking its regular neighborhood to a smooth normal disk bundle. Hence by Theorem 1 of Massey [8] we have that the regular neighborhood of $P$, denoted $\nu(P)$, is homeomorphic to $\nu\left(P^{2}\right)$. Let $h:\left(\nu\left(P^{2}\right), P^{2}\right) \rightarrow(\nu(P), P)$ be a PL homeomorphism. Clearly $h(a)$ is null homotopic in $\nu(P)$. Since $H_{1}\left(S^{4}-\right.$ int $\nu(P) ; Z) \approx Z_{2}$ and since $h(a)$ is not null homologous in $S^{4}-P$, we have either $h(b)$ or else $h(a b)$ null homologous in $S^{4}-\operatorname{int} \nu(P)$. It is easy to construct a homeomorphism of $\nu\left(P^{2}\right)$ onto itself that takes $a$ to $a$ and takes $b$ to $a b$ (see Price [9]). Hence we can assume that $h:\left(\nu\left(P^{2}\right), P^{2}\right) \rightarrow(\nu(P), P)$ is chosen so that $h(b)$ is null homologous in $S^{4}$ - int $\nu(P)$. Finally we assume that $P$ is isotoped so that $P \cap B_{0}^{4}$ is a disk with $\left(B_{v}^{1}, P \cap B_{0}^{1}\right)$ unknotted and that $h$ is chosen so that $h\left(\nu\left(D^{2}\right)\right)=\nu(P) \cap B_{0}^{4}$ and $h\left((\nu) M^{2}\right)=\nu(P) \cap B^{4}$.

Using the above notation we show the following:

LEMmA 1. The homeomorphism $h^{-1} \mid: \nu(P) \cap B^{4} \rightarrow \nu\left(M^{2}\right)$ extends to a $\operatorname{map} f: B^{4} \rightarrow B^{4}$ with

$$
f\left(B^{4}-\operatorname{int} \nu(P)\right) \cong B^{4}-\operatorname{int} \nu\left(P^{2}\right) .
$$

Proof. We will use elementary obstruction theory, see $\mathrm{Hu}$ [6] Chapter VI. First note that $B^{4}-\operatorname{int} \nu\left(P^{2}\right)$ is a deformation retract of $S^{4}-P^{2}$ (since) $\left.B_{0}^{4}, P^{2} \cap B_{0}^{4}\right)$ is unknotted). Hence we have $\pi_{2}\left(B^{4}-\right.$ 
int $\left.\nu\left(P^{2}\right)\right) \approx \pi_{i}\left(S^{4}-P^{2}\right) \approx \pi_{i}\left(P^{2}\right)$. Let $X=B^{4}-\operatorname{int} \nu(P)$ and let $X_{0}=$ $\nu(P) \cap X$. Then $X$ is a deformation retract of $S^{4}-P$ and hence we have $H^{i}(X ; G) \approx H^{i}\left(S^{4}-P ; G\right) \approx H^{i}\left(P^{2}, G\right)$ for any coefficient group, $G$. Similarly, it is easy to check that $X_{0}$ deformation retracts to the Klein bottle $h\left(K^{2}\right)$ and hence $H^{i}\left(X_{0}, G\right) \approx H^{i}\left(K^{2}, G\right)$ for any coefficient group, $G$. The long exact sequence for the pair $\left(X, X_{0}\right)$ yields the following computations.

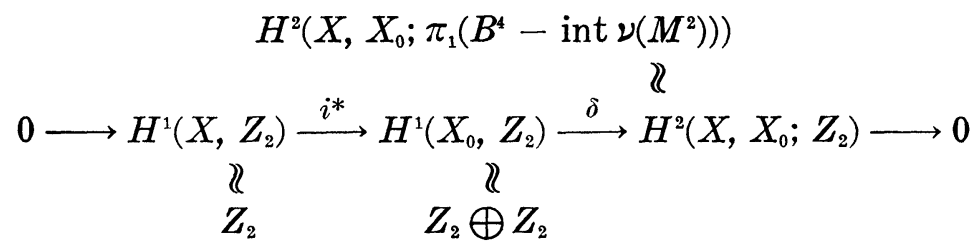

Furthermore, if we let $\bar{a}$ and $\bar{b}$ denote the cocycles algebraically dual to the cycles $h(a)$ and $h(b)$, in $h\left(K^{2}\right) \subseteq X_{0}$, then $H^{1}\left(X, Z_{2}\right)$ is generated by $\bar{a}, H^{1}\left(X_{0}, Z_{2}\right)$ is generated by $\bar{a}$ and $\bar{b}$ and hence $H^{2}\left(X, X_{0} ; Z_{2}\right)$ is generated by $\delta(\bar{b})$. (By algebraically dual we mean that $\langle\bar{a}, h(a)\rangle=1,\langle\bar{a}, h(b)\rangle=0,\langle\bar{b}, h(a)\rangle=0$ and $\langle\bar{b}, h(b)\rangle=1$ where $\langle$,$\rangle denotes the Kronecker product. See Greenberg [4] page 132.)$

$$
\begin{aligned}
& H^{3}\left(X, X_{0} ; \pi_{2}\left(B^{4}-\operatorname{int} \nu\left(M^{2}\right)\right)\right) \\
& \text { 《) } \\
& 0=H^{2}\left(X_{0} ; Z\right) \longrightarrow H^{3}\left(X, X_{0} ; Z\right) \longrightarrow H^{3}(X ; Z)=0 \\
& H^{4}\left(X, X_{0} ; \pi_{3}\left(B^{4}-\operatorname{int} \nu\left(M^{2}\right)\right)\right) \\
& \text { ? } \\
& 0=H^{3}\left(X_{0} ; Z\right) \longrightarrow H^{4}\left(X, X_{0} ; Z\right) \longrightarrow H^{4}(X ; Z)=0
\end{aligned}
$$

From the above computations it is clear that the only possible nonzero obstruction to extending $h^{-1} \mid X_{0}: X_{0} \rightarrow B^{4}-\operatorname{int} \nu\left(M^{2}\right)$ is the 2dimensional obstruction lying in $H^{2}\left(X, X_{0} ; \pi_{1}\left(B^{4}-\operatorname{int} \nu\left(M^{2}\right)\right)\right) \approx Z_{2}$. This obstruction will be zero, if we extend $h^{-1}$ to the 1-skeleton of $X$ in the right way. Towards this end, let $S$ be an orientable surface in $B^{4}-\operatorname{int} \nu(P)$ with $\partial S=h(b)$ (recall that the notation was chosen so that $h(b)$ was null homologous in $S^{4}-\operatorname{int} \nu(P)$ and hence in $\left.B^{4}-\operatorname{int} \nu(P)\right)$. Let $K$ be a triangulation of $X$ that subdivides $X_{0}$ and $S$. Since $b$ bounds a disk in $B^{4}-\operatorname{int} \nu\left(M^{2}\right)$, it is easy to extend $h^{-1} \mid X_{0}$ to take $S$ onto that disk. The extension to the remainder of the 1-skeleton of $K$ may be chosen arbitrarily. Let $g$ denote this extension. The obstruction to extending $g\left|X_{0}=h^{-1}\right| X_{0}$ to the 2-skeleton of $K$, denoted $\gamma^{2}(g)$, lies in $H^{2}\left(X, X_{0} ; \pi_{1}\left(B^{4}-\right.\right.$ int $\left.\left.\nu\left(M^{2}\right)\right)\right) \approx Z_{2}$ which is generated by $\delta(\bar{b})$. Hence to see that $\gamma^{2}(g)=0$ we need only show that $\gamma^{2}(g) \neq \delta(\bar{b})$. To see this, consider $c_{2}(S)$, the fundamental homology class in $H_{2}\left(X, X_{0} ; Z_{2}\right)$ carried by $S$. 
Then $c_{2}(S)$ generates $H_{2}\left(X, X_{0} ; Z_{2}\right) \approx Z_{2}$ and $\left\langle\gamma^{2}(g), c_{2}(S)\right\rangle=0$ because $g(S) \subseteq$ disk (see the definition of $\gamma^{2}(g)$, page $176 \mathrm{Hu}$ [6]). On the other hand $\left\langle\delta(\bar{b}), c_{2}(S)\right\rangle=\left\langle\bar{b}, \partial c_{2}(S)\right\rangle=\langle\bar{b}, b\rangle=1$. Hence $\gamma^{2}(g) \neq \delta(\bar{b})$ and hence $h^{-1} \mid X_{0}$ extends to take the 2-skeleton of $K$ into $B^{4}-$ int $\nu\left(M^{2}\right)$. As remarked before, extending $h^{-1}$ to the 3 - and 4-skeletons of $K$ follows easily because the cohomology groups containing the appropriate obstruction elements are trivial.

Theorem 2. Let $P$ be a $P L$ locally flat projective plane in $S^{4}$. Then $P$ bounds a 3-manifold with singular points having exactly one singular point.

Proof. The idea of the proof is to use the inverse image of the $v * M^{2}$ bounded by $P^{2}$. Of course, we do not have a map of $S^{4}-P$ to $S^{4}-P^{2}$ so the proof is slightly more complicated. Let $R$ be a polyhedron in $S^{4}$ with $R$ PL homeomorphic to $v * M^{2}$ and $P^{2}=\partial R$. We assume further that $R \cap\left(B^{4}-\operatorname{int} \nu\left(M^{2}\right)\right)$ is a solid Klein bottle $K$ with $M_{0}=K \cap \partial \nu\left(M^{2}\right)$ and $M_{1}=K \cap \partial B^{4}$ being Moebius bands. It is easy to see (just as in Theorem 1 ) how to construct such a polyhedron $R$ using Fig. 1. The meridianal disks of $K$ are flat-shaped disks whose boundary consists of an arc parallel to a spanning are of $M^{2}$ plus a straight line interval in $\partial B^{4}$. We also assume that $K$ has a normal bundle in $B^{4}-\operatorname{int} \nu\left(M^{2}\right)$.

We can now apply Theorem 3.3.1 of Williamson [15] to homotope the map $f: B^{4}-$ int $\nu(P) \rightarrow B^{4}-$ int $\nu\left(M^{2}\right)$ given by Lemma 1 , to a map $g$ that agrees with $f$ on $X_{0}$ and $g$ is transverse regular to $K$. In particular, $g^{-1}(K)$ will be a compact 3 -manifold-with-boundary properly embedded in $B^{4}-\operatorname{int} \nu(P)$. The boundary of $g^{-1}(K)$ will consist of $f^{-1}\left(M_{0}\right)=h\left(M_{0}\right)$; a 2-manifold-with-boundary, denoted $M_{1}^{\prime} \subseteq$ $\left(\partial B^{4}\right)$ - int $\nu(P)$ (whose boundary coincides with $\left.\partial h\left(M_{0}\right)\right)$; plus a collection of pairwise disjoint compact 2-manifolds without boundary in $\left(\partial B^{4}\right)$ - int $\nu(P)$, call them $S_{1}, S_{2}, \cdots, S_{n}$.

To complete the construction of the 3 -manifold with singular points bounded by $P$, we merely extend $g^{-1}(K)$ across $B^{4} \cap \nu(P)$ so it contains $h\left(M^{2}\right)$ on its boundary, then we cone over $M_{1}^{\prime}$ (actually we cone over the slightly extended $M_{1}^{\prime}$ ) and finally we cap off each of $S_{1}, S_{2}, \cdots, S_{n}$ with a 3-manifold-with-boundary in $B_{0}^{4}$ - (cone over $\left.M_{1}^{\prime}\right)$ note that each $S_{\imath}$ bounds a 3 -manifold in $\left(\partial B^{4}\right)-\operatorname{int} \nu(P)$ so we can cap off the $S_{2}$ by pushing such a manifold slightly into $B_{0}^{4}$ to keep them pairwise disjoint. The one singular point is introduced when we cone over $M_{1}^{\prime}$.

Note. Dennis Roseman has suggested that Theorem 2 could also be proved using the projection of $P$ into $S^{3}$ combined with a checker- 
board technique analogous to that used for constructing spanning surfaces of knots in $E^{3}$. Using this method of proof, though, one would get one singular point of the 3-manifold with singular points at each branch point of the projection.

In proving Theorem 2 we have avoided trying to map $S^{4}-P$ onto $S^{4}-P^{2}$. Theorem 2 would certainly follow from the existence of such a map and one might hope that the existence of the spanning 3 -manifold with singular points would permit the construction of such a map. All of our attempts to find such a map seem to run into the same difficulty, namely, some loop in the spanning 3manifold with singular points might link $P$ (that is, it might not be null homologous in $S^{4}-P$ ). In Theorem 3 we introduce a hypothesis on $P$ that permits us to construct a spanning 3-manifold with singular points in which no loop links $P$. Then we can map $S^{4}-P$ onto $S^{4}-P^{2}$. The hypothesis we use is that the longitude on $\partial \nu(P)$ bounds a surface $S$, in $S^{4}-$ int $\nu(P)$, so that no loop on $S$ links $P$. Using this surface $S$ one can then construct a "nicer" spanning 3-manifold with singular points than we did in Theorem 2 . (This is not exactly how the proof is written up but it is the reason that the map can now be constructed.) While this new hypothesis is necessary and sufficient for the existence of the desired map, we have two objections to it. The first is that we do not know of an example that fails to have this property in fact we do not even have any strong feeling as to whether or not all projective planes in $S^{4}$ satisfy it. The second objection is that the hypothesis is too geometric in nature and two difficult to verify or disprove. Towards improving this we suggest the following conjecture:

Conjecture. Let $P, h$ and $b$ be as in Theorem 3. Then $h^{-1}$ extends to a map $F: S^{4} \rightarrow S^{4}$ with $F\left(S^{4}-\nu(P)\right) \subseteq S^{4}-\nu\left(P^{2}\right)$ if and only if $h(b)$ lies in the second commutator subgroup of $\pi_{1}\left(S^{4}\right.$. int $\nu(P))$.

It is clear that if $h(b)$ bounds a surface $S$, as in Theorem 3, then $h(b)$ lies in the second commutator subgroup of $\pi_{1}\left(S^{4}-\right.$ int $\left.\nu(P)\right)$. Our attempts at constructing $S$ given the algebraic conditions on $h(b)$ have not succeeded although they come so closure it seems the conjecture must be true.

THEOREM 3. Let $P$ be a $P L$ locally flat projective plane in $S^{4}$. Let $h:\left(\nu\left(P^{2}\right), P^{2}\right) \rightarrow(\nu(P), P)$ be a $P L$ homeomorphism. Let $b$ denote the standard longitude on $\partial \nu\left(P^{2}\right)$. Then $h^{-1}$ extends to a map $F$ : $S^{4} \rightarrow S^{4}$ with $F\left(S^{4}-\nu(P)\right) \subseteq S^{4}-\nu\left(P^{2}\right)$ if and only if $h(b)$ bounds $a$ $P L$ locally flat orientable spanning surface, $S$, in $S^{4}-$ int $\nu(P)$ with $H_{1}(S ; Z) \stackrel{\subseteq}{\rightrightarrows} H_{1}\left(S^{4}-\operatorname{int} \nu(P) ; Z\right)$ being the zero homomorphism. 
Proof. Suppose $h^{-1}$ extends to such a map $F$. Let $D$ be a PL locally flat spanning disk of $S^{4}-\operatorname{int} \nu\left(P^{2}\right)$ with $\partial D=b$. We can assume that $F$ is in general position relative to $D$. Then $F^{-1}(D)$ contains a surface, $S$, with $h^{-1}(b)=\partial S$. Furthermore, since $H_{1}\left(S^{4}-\right.$ int $\left.\nu(P) ; Z\right)$ is generated by a meridian of $\partial \nu(P)$ and since $h^{-1}$ takes meridians of $\partial \nu(P)$ to meridians of $\partial \nu\left(P^{2}\right)$, it follows that

$$
H_{1}(S ; Z) \stackrel{\subseteq}{\longrightarrow} H_{1}\left(S^{4}-\operatorname{int} \nu(P) ; Z\right)
$$

is the zero homomorphism.

Conversely, suppose $S$ is a surface satisfying the above hypothesis. Let $N(S)$ be a regular neighborhood of $S$ in $S^{4}-\operatorname{int} \nu(P)$. As above, let $D$ be a PL locally flat spanning disk of $S^{4}-\operatorname{int} \nu\left(P^{2}\right)$ with $\partial D=b$. Let $N(D)$ be a regular neighborhood of $D$ in $S^{4}$ int $\nu\left(P^{2}\right)$. We assume further that $D$ is chosen so that $\nu\left(P^{2}\right) \cup N(D)$ is a regular neighborhood of an unknotted 2-sphere in $S^{4}$ (in Fig. 1 replace a narrow Moebius band about the centerline of $M^{2}$ by the cone over its boundary, taken in $N(D)$ ). It follows then that closure $\left(S^{4}-\left(\nu\left(P^{2}\right) \cup N(D)\right)\right)$ is homeomorphic to $S^{1} \times B^{3}$.

Both $N(S)$ and $N(D)$ are products and we identify them with $S \times D^{2}$ and $D \times D^{2}$ via some fixed homeomorphism. Using this product structure it is easy to extend $h^{-1}$ to a map $f: \nu(P) \cup$ $N(S) \rightarrow \nu\left(P^{2}\right) \cup N(D)$. We assume further that $h^{-1}$ was isotoped so that $f$ can be chosen with $f \mid N(S): N(S) \rightarrow N(D)$ a product map taking $S$ onto $D$ and $s \times D^{2} \subseteq S \times D^{2} \equiv N(S)$ homeomorphically onto (image(s)) $\times D^{2} \subseteq D \times D^{2} \equiv N(D)$. Such an extension is, of course, not unique and a little later we will have to adjust $f$ so it does not rotate these normal disks. In order to extend $f$ further, we resort to obstruction theory. Let $X=S^{4}-\operatorname{int}(\nu(P) \cup N(S))$ and let $Y=S^{4}-\operatorname{int}\left(\nu\left(P^{2}\right) \cup N(D)\right)$. Then $f$ takes $\partial X$ onto $\partial Y \subseteq Y$ and we wish to extend $f$ to take $X$ into $Y$.

As noted above, $Y \approx S^{1} \times B^{3}$, hence $\pi_{1}(Y) \approx Z$ and $\pi_{i}(Y)=0$ if $i>1$. The obstructions to extending $f$ to all of $X$ line in $H^{i+1}\left(X, \partial X ; \pi_{i}(Y)\right)$ which are all zero except for $H^{2}(X, \partial X ; Z)$. To complete the proof of the lemma we show how to extend $f$ to the 1-skeleton of $X$ in such a way that the obstruction in $H^{2}(X, \partial X ; Z)$, to extending to the 2 -skeleton, is zero. To do that we take a careful look at $H_{1}(\partial X ; Z)$ and $H_{1}(X ; Z)$.

First we note that, since $X$ is homotopy equivalent to $S^{4}-$ $(P \cup S)$, we have, by Alexander Duality, $H_{1}(X ; Z) \approx Z \approx\{\alpha: \quad\}$ where $\alpha$ is carried by a simple closed curve in $\partial \nu(P)-N(S)$ normal to $P$ (e.g., $\alpha=h^{-1}(a)$ and $H_{1}(Y ; Z) \approx \pi_{1}(Y) \approx\{a: \quad\}$ where $a$, as usual, is a meridian on $\partial \nu\left(P^{2}\right)$ (we assume that $a$ is isotoped into $\partial \nu\left(P^{2}\right)-$ $N(D))$. Of course, we also have that $f_{*}(\alpha)=a$. 


\section{Clearly $\partial X \approx(\partial \nu(P)-\operatorname{int} N(\partial S)) \bigcup_{\partial} S \times S^{1}$. Furthermore} $H_{1}\left(S \times S^{1}\right)$ is generated by $\gamma_{0}, \cdots, \gamma_{q}$ where $\gamma_{0}$ is carried by point $\times S^{1} \cong S \times S^{1}$ while $\gamma_{1}, \cdots, \gamma_{q}$ are carried by curves in $S \times S^{1}$ that project to "longitudes and meridians" of the handles of $S$. One easily checks that $i_{1}\left(\gamma_{0}\right)=2 \alpha \in H_{1}(X ; Z)$, where $i_{1}: H_{1}\left(S \times S^{1} ; Z\right) \rightarrow$ $H_{1}(X ; Z)$ is induced by inclusion. (See Fig. 1 and think of $\gamma_{0}$ as lying on $\partial \nu$ being normal to $b$.) The next observation we need is that we can assume that $i_{1}\left(\gamma_{j}\right)=0$ for $j>0$. To see this, note that under the composition

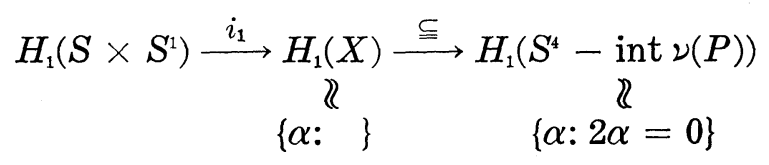

each $\gamma_{j}(j>0)$ goes to zero (by hypothesis). Hence $i_{1}\left(\gamma_{j}\right)=2 n_{j} \alpha$. Now let $\gamma_{j}^{\prime}=\gamma_{j}-\eta_{j} \gamma_{0}$. Then $i_{1}\left(\gamma_{j}^{\prime}\right)=0$ and $\gamma_{j}^{\prime}$ is still carried by a curve that projects to a longitude or a meridian of $S$ (it just "twists" around $S$ differently). To simplify notation we write $\gamma_{j}$ instead of $\gamma_{j}^{\prime}$. We will also need to have $f_{*} i_{1}\left(\gamma_{j}\right)=0 \in H_{1}(\partial Y) \underset{\approx}{\stackrel{\subsetneq}{\leftrightarrows}} H_{1}(Y) \approx\{a: \quad\}$. Since $f$ might not have this property, we use Lemma 2 to modify the map $f$ so it will have this property. From properties of the map $f$, we know that $f_{*} i_{1}\left(\gamma_{j}\right)$ is a multiple, say $m$, of [point $\left.\times \partial D^{2}\right]=$ $2 a$ in $H_{1}(\partial Y)$. Let $T$ be a disk with one handle contained in $S$ which contains the meridian or longitude that $\alpha_{j}$ projects to an interests only one other meridian or longitude corresponding to any $\gamma_{i}$. (We assume that the $\gamma_{i}$ 's were chosen so that they could be separated this way.) Let $g$ be a homeomorphism of $T \times D^{2} \cong N(S)=S \times D^{2}$ onto itself, satisfying the conclusion of Lemma 2 using a carrier of $\gamma_{j}$ for $K_{2}$ and the negative of the integer $m$ determined above. Then $g$ extends to all of $\nu(P) \cup N(S)$ by letting it be the identity outside of $T \times D^{2}$. Furthermore, $g_{*} i_{1}\left(\gamma_{j}\right)=i_{1}\left(\gamma_{j}\right)-m \cdot 2 \alpha$ so $f_{*} g_{*} i_{1}\left(\gamma_{j}\right)=f_{*} i_{1}\left(\gamma_{j}\right)-m \cdot 2 a=0$ while $f_{*} g_{*} i_{1}\left(\gamma_{k}\right)=f_{*} i_{1}\left(\gamma_{k}\right)$ for $k \neq j$. Hence $f \circ g$ acts the right way on $\gamma_{j}$ and does not change the action of $f$ on the other $\gamma_{k}$ 's. Repeated application of this technique provides us with the map, which we still call $f$, from $\nu(P) \cup N(S)$ to $\nu\left(P^{2}\right) \cup N(D)$ satisfying $f_{*} i_{1}\left(\gamma_{j}\right)=0 \in H_{1}(\partial \gamma)(1 \leqq j \leqq q)$. Finally we observe that $H_{1}(\partial \nu(P)-\operatorname{int} N(\partial S) ; Z)$ is generated by cycles $\alpha$ and $\beta$ where $\alpha$ is as above and $\beta$ is carried by a curve in $\partial N(\partial S)$, parallel to $\partial S$ and bounding a surface in $S \times S^{\prime} \cong \partial N(S)$ parallel to $S$. Then $i_{2}(\beta)=0 \in H_{1}(X)$ and $f_{*} i_{2}(\beta)=0 \in H_{1}(Y)$ where $i_{2}$ is induced by including $\partial \nu(P)-\operatorname{int} N(\partial S)$ into $X$.

Now the appropriate part of the Mayer-Vietoris sequence

$$
H_{1}\left(S \times S^{1}\right) \oplus H_{1}(\partial \nu(P)-\operatorname{int} N(\partial S)) \stackrel{\phi_{1}+\dot{\phi}_{2}}{\longrightarrow} H_{1}(\partial X) \longrightarrow 0
$$


yields that an arbitrary element $\eta$ of $H_{1}(\partial X)$ can be written as $\phi_{1}\left(\eta_{1}\right)+\phi_{2}\left(\eta_{2}\right)$ where $\eta_{1} \in H_{1}\left(S \times S^{\prime}\right)$ and $\eta_{2} \in H_{1}(\partial \nu(P)-\operatorname{int} N(\partial S))$. Using the above notation and computations we have that $\eta_{1}=$ $\sum_{i=0}^{q} k_{i} \gamma_{i}$ and $\eta_{2}=m \alpha+n \beta$ for some integers $k_{0}, k_{1}, \cdots, k_{q}, m$ and $n$. Hence we can write $\eta=\sum_{i=0}^{q} k_{i} \phi_{1}\left(\gamma_{i}\right)+m \dot{\phi}_{2}(\alpha)+n \dot{\phi}_{2}(\beta)$.

Let $\phi: H_{1}(\partial X) \rightarrow H_{1}(X)$ be induced by inclusion. Let $\xi \epsilon$ $H_{2}(X, \partial X ; Z)$, and let $\eta=\partial \xi \in H_{1}(\partial X)$. In this case $\phi(\eta)=\phi(\partial \xi)=$ $\sum_{i=0}^{q} k_{i} \dot{\phi} \dot{\phi}_{1}\left(\gamma_{i}\right)+m \dot{\rho} \dot{\phi}_{2}(\alpha)+n \dot{\phi} \dot{\phi}_{2}(\beta)=k_{0} \dot{\phi} \dot{\phi}_{1}\left(\gamma_{0}\right)+m \dot{\rho} \dot{\phi}_{2}(\alpha)=k_{0} \cdot 2 \alpha+m \cdot \alpha$ (we write $\alpha$ for $\phi \dot{\phi}_{2}(\alpha)$ and $i_{2}(\alpha)$, etc.). Of course, by exactness, $\phi(\eta)=0$ so $2 k_{0}+m=0$. With $\xi$ as above we have $f_{*}\left(\hat{o}_{\xi}\right)=$ $\sum_{i=0}^{q} k_{\imath} f_{*} \dot{\phi}_{1}\left(\gamma_{i}\right)+m f_{*} \dot{\phi}_{2}(\alpha)+n f_{*} \dot{\phi}_{2}(\beta)=k_{0} f_{*} \dot{\rho}_{1}\left(\gamma_{0}\right)+m f^{*} \dot{\rho}_{2}(\alpha)=k_{0} .2 a+$ $m a=0$ because $2 k_{0}+m=0$.

Finally we are in a position to extend $f$. Let $\Sigma_{1}, \Sigma_{2}, \cdots, \nu_{p}$ be orientable spanning surfaces of $X$ so that $\left[\Sigma_{1}\right],\left[\Sigma_{2}\right], \cdots,\left[\Sigma_{p}\right]$ generate $H_{2}(X, \partial X ; A)$. Applying the previous paragraph to $\partial\left[\Sigma_{i}\right] \in H_{1}(\partial X)$ we have $f_{*}\left(\partial\left[\Sigma_{i}\right]\right)=0 \in H_{1}(\partial Y) \stackrel{\subseteq}{\underset{ }{\approx}} H_{1}(Y)$. Hence it is easy to extend $f$ to take $\Sigma_{i}$ into $Y$. The $\Sigma_{i}$ 's need not be pairwise disjoint but by general position we can assume that they intersect in at most a finite collection of points in their interiors. It is easy to prove that $f: \nu(P) \cup N(S) \rightarrow \nu\left(P^{2}\right) \cup N(D)$ extends to take $\bigcup_{i=1}^{n} \Sigma_{i}$ into $Y$. Since $Y$ is path connected this map extends further to take the 1-skeleton of some triangulation of $X$ into $Y$. Call this extension $f_{1}$. Then the obstruction to extending $f_{1} \mid \partial X: \partial X \rightarrow Y$ to take the 2-skeleton of $X$ into $Y$ lies in $H^{2}\left(X, \partial X ; \pi_{1}(Y)\right)$. Since $f_{1}$ is already defined on $\Sigma_{1}, \Sigma_{2}, \cdots$ and $\Sigma_{p}$ and since their homology classes generate $H_{2}(X, \partial X ; Z)$ it is easy to check (from the definition of the obstruction cocycle) that the obstruction is, in fact, zero. Hence the map does extend to the 2-skeleton and as we remarked earlier it extends to the 3 -skeleton and 4 -skeleton by virtue of the fact that those obstructions lie in trivial cohomology groups.

LemMa 2. Let $T$ be a disk with one handle. Let $J_{1}$ and $J_{2}$ be a pair of simple closed curves on $T$ that intersect transversely in exactly one point. Let $K_{1}$ and $K_{2}$ be simple closed curves on $\partial\left(T \times D^{2}\right)$ such that projection from $T \times D^{2}$ to $T$ takes $K_{i}$ homeomorphically onto $J_{i}(i=1,2)$. Let $m$ be an integer. Then there exists a homeomorphism $h: T \times D^{2} \rightarrow T \times D^{2}$ satisfying

(a) $h \mid(\partial T) \times D^{2}=$ identity,

(b) $h(T \times 0)=T \times 0$,

(c) $h \mid K_{1}=$ identity,

(d) $h\left(K_{2}\right)$ projects onto $J_{2}$, and

(e) $\left[h\left(K_{2}\right)\right]=\left[K_{2}\right]-m$. $\left[\right.$ point $\left.\times \partial D^{2}\right] \in H_{1}\left(T \times \partial D^{2} ; Z\right)$.

Proof. This is a standard twisting type homeomorphism. Let 
$A$ be an annulus in $T$ with $A$ parallel to $J_{1}$, but $A \cap J_{1}=\dot{\phi}$ and $A \cap J_{2}$ being an arc. We consider $A$ as a family of simple closed curves $\left\{A_{t}\right\}_{t \in[0,1]}$. The homeomorphism $h$ will be the identity except on $A \times D^{2}$. For each $x \in A_{t}$, h takes $x \times D^{2}$ onto itself by a rotation of $-2 \pi m t$ radians. Hence $h$ is the identity on $A_{0} \times D^{2}$ and $A_{1} \times D^{2}$. The curve $K_{2}$ misses $A \times D^{2}$ so $h \mid K_{2}=$ identity. The curve $K_{1}$ intersects $A \times D^{2}$ in an arc lying over $J_{1} \cap A$ and the arc, $h\left(K_{1} \cap\left(A \times D^{2}\right)\right)$ now "wraps around" $\left(J_{1} \cap A\right) \times D^{2}$ exactly $m$ times fewer than $K_{1} \cap\left(A \times D^{2}\right)$ did, hence (e) follows. The other conclusions are obvious.

\section{REFERENCES}

1. M. M. Cohen, A general theory of relative regular neighborhoods, Trans. Amer. Math. Soc., 136 (1969), 189-230.

2. R. H. Crowell, and R. H. Fox, Introduction to Knot Theory, Ginn and Co., 1963.

3. R. A. Fox and J. W. Milnor, Singularities of 2-spheres in 4-space and eigenvalence of knots, Bull. Amer. Math. Soc., 63 (1957), 406.

4. M. J. Greenberg, Lectures on Algebraic Topology, W. A. Benjamin, Inc., 1967.

5. M. W. Hirsch, Smooth regular neighborhoods, Ann. Math., 76 (1962), 524-530.

6. S. T. Hu, Homotopy Theory, Academic Press, 1959.

7. J. F. P. Hudson, Piecewise Linear Topology, W. A. Benjamin Inc., 1969.

8. W. S. Massey, Imbeddings of projective planes and related manifolds in spheres, Ind. Math. J., 23 (1974), 791-812.

9. T. M. Price, Homeomorphisms of quaternion space and projective planes in four space. J. Austral. Math. Soc.

10. T. M. Price and D. M. Roseman, Embeddings of the projective plane in four space, submitted.

11. H. Schubert, Knoten und vollringe, Acta Math., 90 (1953), 131-286.

12. H. Terasaka, On null equivalent knots, Osaka Math. J., 11 (1959), 95-113.

13. C. T. C. Wall, Locally flat PL submanifolds with codimension two, Proc. Cambridge Philos. Soc., 63 (1967), 5-8.

14. H. Whitney, Differentiable Manifolds, Lectures in Topology, ed. by W. L. Ayres and R. L. Wilder, Univ. of Michigan Press, 1941.

15. R. E. Williamson, Cobordism of combinatorial manifolds, Ann. of Math., 83 (1966), 1-33.

Received January 31, 1977 and in revised form September 30, 1977.

UNIVERSITY OF IOWA

IOWA CITY, IA

AND

UNIVERSITY OF AUCKLAND

Auckland, New Zealand 



\section{PACIFIC JOURNAL OF MATHEMATICS}

\section{EDITORS}

DONALD BABBITT (Managing Editor)

University of Galifornia

Los Angeles, California 90024

Hugo RossI

University of Utah

Salt Lake City, UT 84112

C. C. MoOre AND ANDrew OGG

University of California

Berkeley, CA 94720

\section{J. DugundjI}

Department of Mathematics University of Southern California

Los Angeles, California 90007

R. Finn and J. Milgram

Stanford University

Stanford, California 94305

ASSOCIATE EDITORS

E. F. Beckenbachi

B. H. NeumanN

F. WOLF

K. YOSHIDA

\section{SUPPORTING INSTITUTIONS}

UNIVERSITY OF BRITISH COLUMBIA

CALIFORNIA INSTITUTE OF TECHNOLOGY

UNIVERSITY OF CALIFORNIA

MONTANA STATE UNIVERSITY

UNIVERSITY OF NEVADA, RENO

NEW MEXICO STATE UNIVERSITY

OREGON STATE UNIVERSITY

UNIVERSITY OF OREGON
UNIVERSITY OF SOUTHERN CALIFONIA

STANFORD UNIVERSITY

UNIVERSITY OF HAWAII

UNIVERSITY OF TOKYO

UNIVERSITY OF UTAH

WASHINGTON STATE UNIVERSITY

UNIVERSITY OF WASHINGTON

Printed in Japan by International Academic Printing Co., Ltd., Tokyo, Japan 


\section{Pacific Journal of Mathematics \\ Vol. 89 , No. 1 \\ May, 1980}

David Bressoud, A note on gap-frequency partitions ................ 1

John David Brillhart, A double inversion formula ................. 7

Frank Richard Deutsch, Günther Nürnberger and Ivan Singer, Weak

Chebyshev subspaces and alternation .......................

Edward Richard Fadell, The relationship between Ljusternik-Schnirelman

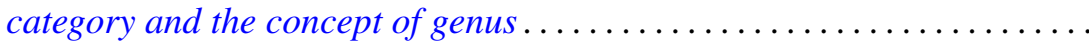

Harriet Jane Fell, On the zeros of convex combinations of polynomials.......

John Albert Fridy, An addendum to: "Tauberian theorems via block

dominated matrices" ..................................

Andrzej Granas, Ronald Bernard Guenther and John Walter Lee, Applications of topological transversality to differential equations. I. Some nonlinear

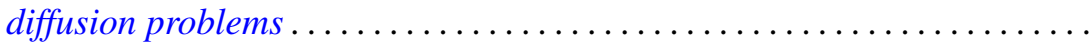

David E. Handelman and G. Renault, Actions of finite groups on self-injective

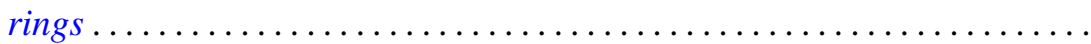

Michael Frank Hutchinson, Local $\Lambda$ sets for profinite groups .............

Arnold Samuel Kas, On the handlebody decomposition associated to a

Lefschetz fibration...

Hans Keller, On the lattice of all closed subspaces of a Hermitian space.....

P. S. Kenderov, Dense strong continuity of pointwise continuous

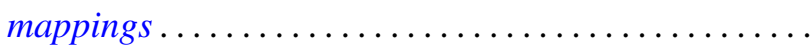

Robert Edward Kennedy, Krull rings.................

Jean Ann Larson, Richard Joseph Laver and George Frank McNulty,

Square-free and cube-free colorings of the ordinals ...

Viktor Losert and Harald Rindler, Cyclic vectors for $L^{p}(G)$

John Rowlay Martin and Edward D. Tymchatyn, Fixed point sets of

1-dimensional Peano continua...

Augusto Nobile, On equisingular families of isolated singularities ...

Kenneth Joseph Prevot, Imbedding smooth involutions in trivial bundles ...

Thomas Munro Price, Spanning surfaces for projective planes in four space.

Dave Riffelmacher, Sweedler's two-cocycles and Hochschild cohomology....

Niels Schwartz, Archimedean lattice-ordered fields that are algebraic over

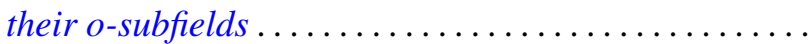

Chao-Liang Shen, A note on the automorphism groups of simple dimension groups.

Kenneth Barry Stolarsky, Mapping properties, growth, and uniqueness of

Vieta (infinite cosine) products ...

Warren James Wong, Maps on simple algebras preserving zero products. I.

The associative case ............................. 